\title{
Cephalic Arch Restenosis Rates in Hemodialysis Patients with Brachiocephalic Fistulae: A Retrospective Multicenter Analysis of 3,301 Patients
}

Rishi N. Razdan ( $\sim$ Rishi.Razdan@AzuraCare.com )

Azura Vascular Care

Melvin Rosenblatt

Azura Vascular Care

Yue Jiao

Fresenius Medical Care (United States)

Nancy McLaughlin

Azura Vascular Care

Len Usvyat

Fresenius Medical Care (United States)

Murat Sor

Azura Vascular Care

John Larkin

Fresenius Medical Care (United States)

\section{Research Article}

Keywords: Cephalic arch, stenosis, brachiocephalic fistula, arteriovenous fistula, end stage renal disease, angioplasty, stent

Posted Date: September 20th, 2021

DOl: https://doi.org/10.21203/rs.3.rs-909183/v1

License: (c) (1) This work is licensed under a Creative Commons Attribution 4.0 International License. Read Full License 


\section{Abstract \\ Background:}

We evaluated restenosis rates at the cephalic arch after percutaneous angioplasty and stenting procedures in patients with brachial artery to cephalic vein arteriovenous fistula (BCAVF) hemodialysis access.

\section{Methods:}

We used data from adult hemodialysis patients treated at a national network of 44 outpatient interventional facilities during Oct 2011-2015. We included data from patients with BCAVF who received an exclusive angioplasty, or stent with angioplasty, for treatment of cephalic arch stenosis and had $\geq 1$ subsequent evaluation of the cephalic arch. Median percent restenosis per month at cephalic arch and days between encounters was calculated from the $1 \mathrm{st}$ index to 2 nd procedure, and for up to 4 subsequent encounters. Analyses were stratified by intervention and device types.

\section{Results:}

We identified a cohort of 3,301 patients (mean age $62.2 \pm 13.9$ years, $58.5 \%$ male, $33.2 \%$ white race) with a BCAVF who had an angioplasty, or stent, at the cephalic arch for an index and $\geq 1$ follow-up procedure. Between the 1 st index to 2 nd procedure, patients who received an angioplasty $(n=2,663)$ or stent $(n=$ 933) showed a median decrease of $18.9 \%$ and $16.5 \%$ in luminal diameter per month and a median time of 93 and 91 days between encounters, respectively. Restenosis and day rates were similar for standard versus high-pressure angioplasties. Bare metal stents showed 10.1 percentage point higher restenosis rate compared to stent grafts. Restenosis rates and time to restenosis were relatively consistent across subsequent encounters.

\section{Conclusions:}

Findings suggest hemodialysis patients with a BCAVF who require an angioplasty or stent to treat a stenosis at the cephalic arch will have stenosis reformed at a rate of $18.9 \%$ and $16.5 \%$ per month after the first intervention, respectively. Findings suggest patients are at risk of having significant lesions at the cephalic arch within 3 months after the previous intervention.

\section{Background:}

In the United States, more than 785,000 people are diagnosed with kidney failure and require kidney replacement therapy (KRT) to sustain life.[1] About $62 \%$ of patients are treated by the KRT of hemodialysis, whereby blood is filtered three or more times per week using a vascular access. Dialysis 
access types include arteriovenous fistulae (AVFs), arteriovenous grafts (AVGs), or catheters. Of these options, AVFs are considered the preferred access given better function and lower infection and mortality rates compared to catheters and AVGs.[2] Also, studies have shown that AVFs demonstrate lower thrombosis and access failure rates than AVGs.[3] Among prevalent hemodialysis patients, about $66 \%$ use an AVF.[1]

The three most common AVFs are the radio-cephalic fistula, brachial artery to cephalic vein fistula, and brachial artery to basilic vein transposed fistula. Although the first choice is the radio-cephalic forearm fistula, the brachial artery to cephalic vein fistula, or brachiocephalic fistula (BCAVF), is one of the most popular types for many reasons. Among several advantages is ease of creation, high maturation rates, and high flow rates. Disadvantages include higher rates of steal syndrome and symptomatic central venous stenosis.[4]

With an increasing prevalence of BCAVF comes unique complications requiring treatment to avoid failure. For BCAVF, the typical site of stenosis is the cephalic arch.[4-7] Potential etiologies for stenosis and subsequent restenosis at the cephalic arch include increased flow in a fistulized vein, external compression by fascia and pectoralis major, angulation, numerous valves in the outflow vein, and biochemical changes associated with kidney failure. The cephalic arch is typically a single channel joining with the axillary vein, yet variant anatomy such as a bifid arch, or abnormal termination point (internal or external jugular veins), can cause venous outflow obstruction and access malfunction.[8] It is likely a combination of multiple factors contribute to occurrence and recurrence of stenosis at this site.

To date, there are no large multicenter studies describing the treatment and effectiveness of percutaneous interventions at the cephalic arch in BCAVFs. Our study aimed to evaluate cephalic arch restenosis rates after percutaneous intervention, including transluminal balloon angioplasty and stenting.

\section{Methods:}

\section{Setting:}

We used data from a network of 44 outpatient vascular care and ambulatory surgery centers (Azura Vascular Care, Malvern, PA) across the United States between October-2011 through October-2015. This analysis was conducted under a protocol approved by New England Independent Review Board (IRB). The IRB determined this analysis of deidentified patient data was exempt and did not require informed consent per the United States Code of Federal Regulations 45CFR46 (Needham Heights, MA; NEIRB\#WO1-574-1). This study adhered with the Declaration of Helsinki.

\section{Patient Population:}

We included data from adult patients (age $\geq 18$ years) with a brachiocephalic AVF (BCAVF) who: 1) were referred for evaluation of an access malfunction, 2) received an angioplasty or stent for treatment of stenosis at the cephalic arch, and 3) had $\geq 1$ subsequent evaluation of the cephalic arch. After the initial 
referral, subsequent encounters were either a clinically timed evaluation scheduled by the interventionalist, or another referral for an access malfunction (e.g. prolonged bleeding, high venous pressures, low access flows). We excluded data from patients who received a coinciding thrombectomy, pharmacomechanical thrombolysis, or embolization at the same encounter as an angioplasty/stent.

\section{Standard of Care Practices:}

Patient care was performed under the provider's standard operating procedures by interventional radiologists, interventional nephrologists, and vascular surgeons who specialize in dialysis access care. A universal electronic medical record system was used to record encounters/procedures. Patients referred for evaluation of a malfunctioning AVF had historical and physical examination performed prior to intervention. Intravenous fentanyl and midazolam were used for moderate sedation under nurse/anesthesiologist monitoring; propofol was also administered in rare instances. A combination of fluoroscopy with contrast and ultrasound was used for evaluation of fistulae. Percutaneous intervention(s) were performed on stenoses $>50 \%$.

Standard practice was typically to treat detected lesions with standard angioplasty ( $<24$ atmospheres (ATM) rated burst pressure balloon) until stenosis was eliminated using inflation times based on operator discretion. When necessary, high-pressure angioplasty (>24 ATM rated burst pressure balloon) were recommended to eliminate a lesion if resistant to standard angioplasty, or a known history of a lesion at the site requiring high-pressure angioplasty. Balloon size ranged from 4-to-14 $\mathrm{mm}$ in diameter. If a residual stenosis was identified, or if a stenosis was unresponsive to angioplasty, a stent or stent graft was placed. Stent size was recommended to be based upon comparison of the normal appearing vein with oversizing by $20-40 \%$ to accommodate for vein elasticity and position the stent. A post-stent deployment angioplasty could be performed to further expand the stent as clinically indicated.

\section{Cephalic Arch Stenosis:}

Cephalic arch was defined as the portion of the cephalic vein within $5 \mathrm{~cm}$ of the confluence with the axillary vein, or larger outflow vein in instances of aberrant anatomy. Stenoses were assessed comparing the narrowest luminal diameter at the cephalic arch, as a percentage of the nearest normal appearing vein. Percent stenosis was documented before and after each intervention.

\section{Analysis Design:}

\section{Analysis of Restenosis Rates by Intervention Type:}

We assessed data from patients who received either exclusively an angioplasty, or a stent with an angioplasty, for treatment of stenosis at the cephalic arch at each index procedure. The first encounter defined the 1 st index procedure for patients who had an angioplasty without any stent placement. The first stent placement defined the 1 st index procedure for patients who received a stent with an angioplasty. 
For each intervention type (angioplasty or stent group), we calculated the per patient difference in the percent stenosis after the 1 st index procedure (post-intervention) to the percent stenosis before the 2 nd procedure (pre-intervention). We computed restenosis rates via the change in the percent stenosis per month from the 1 st index to 2 nd procedure. Also, we determined the days between procedures. Consistent calculations were made between subsequent encounters (2nd index to $3 \mathrm{rd}$, $3 \mathrm{rd}$ index to 4 th procedure). Rates were reported as median value and lower (25\%) and upper (75\%) confidence limit (CL).

Within the angioplasty group defined at the 1 st index procedure, restenosis rates were calculated for the sub-group who had an exclusive angioplasty at the 2nd index to 3rd, as well as the 3rd index to 4th, procedures. Among the stent group, restenosis rates were calculated for the sub-groups who received an angioplasty without any stent at the subsequent 2 nd index to 3 rd and 3rd index to 4th procedures, or another stent at the subsequent 2nd index to 3rd and 3rd index to 4th procedures.

\section{Analysis of Restenosis Rates by Intervention and Device Types:}

We stratified the analysis for each intervention type by the device type used across visits. For angioplasty group, device types were classified as standard or high-pressure. For stent group, device types were classified as bare metal and stent graft (covered stent).

We further assessed the subset who received an index stent at the 1st visit, and a subsequent exclusive angioplasty at the 2 nd index and/or 3rd index visits. For this, we evaluated restenosis rates overall and by device type of the stent at the 1st index visit, as well as the device type of the angioplasty at the $2 \mathrm{nd}$ index and 3rd index visits.

\section{Results:}

\section{Patient Characteristics:}

We identified 3,301 eligible patients with a brachiocephalic AVF (BCAVF) who had an exclusive angioplasty, or a stent with an angioplasty, at the cephalic arch for an index procedure and $\geq 1$ follow-up procedure(s) during the 4-years (Fig. 1). Cohort was on average $62.2 \pm 13.9$ years old, $58.5 \%$ male, $33.2 \%$ white race, $30.9 \%$ black race, and $75.9 \%$ had a left AVF (Table 1). On average patients had $5.0 \pm 3.2$ procedures at the cephalic arch across follow-up. 
Table 1

Patient Characteristics

\begin{tabular}{|ll|}
\hline Parameter & $\mathbf{N}(\%)$, Mean \pm SD \\
\hline Eligible Adult Patients & $3,301(100)$ \\
\hline Age years & $62.2 \pm 13.9$ \\
\hline Male & $1,930(58.5)$ \\
\hline Race & \\
\hline White & $1,097(33.2)$ \\
\hline Black & $1,019(30.9)$ \\
\hline Asian & $53(1.6)$ \\
\hline Other & $46(1.4)$ \\
\hline Unknown & $1,086(32.9)$ \\
\hline Ethnicity & \\
\hline Not Hispanic & $1,731(52.4)$ \\
\hline Hispanic & $484(14.7)$ \\
\hline Unknown & $1,086(32.9)$ \\
\hline AV Fistula Lateral Location & \\
\hline Left & $2,504(75.9)$ \\
\hline Right & $785(23.8)$ \\
\hline Unknown & $12(0.4)$ \\
\hline Total Number of Procedures in Cohort & 16,615 \\
\hline Procedures per Patient & $5.03 \pm 3.15$ \\
\hline N: patient number; SD: standard deviation & \\
\hline Rater after & \\
\hline
\end{tabular}

\section{Restenosis Rates after Angioplasty:}

There were 2,663 patients who received an exclusive angioplasty without a stent for treatment of stenosis at the cephalic arch during the $1 \mathrm{st}$ index visit and returned for a 2 nd follow-up visit. Between the $1 \mathrm{st}$ index to 2 nd visit, this group showed a median decrease of $18.9 \%$ in luminal diameter per month and a median time of 93 days between encounters (Table 2). 
Table 2

Cephalic Arch Restenosis Rates Over Time by Intervention Type

\begin{tabular}{|llll|}
\hline Angioplasty without Stent & Visit Pair & & \\
\hline & 1st to 2nd & 2nd to 3rd & 3rd to 4th \\
\hline Change in Luminal Diameter & $\mathrm{n}=2,663$ & $\mathrm{n}=1,513$ & $\mathrm{n}=901$ \\
\hline Median \% Decrease per Month (CL) & $18.9(12.3-30.0)$ & $18.4(12.7-25.1)$ & $17.8(12.9-24.2)$ \\
\hline Median Days between Encounters (CL) & $93(58-128)$ & $96(75-124)$ & 94 (84-119) \\
\hline Stent & Visit Pair & & \\
\hline & 1st to 2nd & 2nd to 3rd & 3rd to 4th \\
\hline Change in Luminal Diameter & $\mathrm{n}=933$ & $\mathrm{n}=48$ & $\mathrm{n}=5$ \\
\hline Median \% Decrease per Month (CL) & $16.5(7.5-27.2)$ & $19.1(14.6-29.1)$ & 19.8 (13.0-24.6) \\
\hline Median Days between Encounters (CL) & $91(42-126)$ & $91(59-115)$ & 95 (93-100) \\
\hline CL: 25-75\% confidence limit & & & \\
\hline
\end{tabular}

A subset of $56.8 \%$ and $33.8 \%$ of patients received a subsequent exclusive angioplasty to treat restenosis at the 2nd index visit and 3rd index visit, respectively. Between the 2nd index to 3rd and 3rd index to 4th visits, patients showed a median decrease of $18.4 \%$ and $17.8 \%$ in luminal diameter per month and a median time of 96 and 94 days between encounters. There were $11.1 \%$ of patients with an exclusive angioplasty at the 1 st index visit who received a stent at the subsequent 2 nd or 3 rd index visit. These patients were included in the stent group as of the index encounter where they received a stent.

Assessment of angioplasty types showed standard balloons were generally utilized. During the $1 \mathrm{st}$ index visit, $18.4 \%$ of angioplasties used a high-pressure balloon and the remainder used standard balloons; high-pressure balloons were used in $22.5 \%$ and $23.8 \%$ of angioplasties at the 2 nd index and 3 rd index visits (Table 3). Restenosis rates were similar between angioplasty types and median days between encounters were the same at the 1 st index to 2 nd visit, yet progressively increased to differ by 5 days more in the standard versus high-pressure angioplasty groups between the 3rd to 4th visit. 
Table 3

Cephalic Arch Restenosis Rates Over Time by Angioplasty Type

\begin{tabular}{|llll|}
\hline Standard Angioplasty & Visit Pair & & \\
\hline & 1st to 2nd & 2nd to 3rd & 3rd to 4th \\
\hline Change in Luminal Diameter & $\mathrm{n}=2,173$ & $\mathrm{n}=1,172$ & $\mathrm{n}=687$ \\
\hline Median \% Decrease per Month (CL) & $18.6(12.2-29.0)$ & $18.4(12.7-25.1)$ & $17.1(12.4-23.3)$ \\
\hline Median Days between Encounters (CL) & $93(60-126)$ & $95(77-121)$ & 96 (85-121) \\
\hline High-Pressure Angioplasty & Visit Pair & & \\
\hline & 1st to 2nd & 2nd to 3rd & 3rd to 4th \\
\hline Change in Luminal Diameter & $\mathrm{n}=490$ & $\mathrm{n}=341$ & $\mathrm{n}=214$ \\
\hline Median \% Decrease per Month (CL) & $20.3(13.1-32.8)$ & $19.3(12.8-28.5)$ & 19.2 (14.2-28.2) \\
\hline Median Days between Encounters (CL) & $93(56-133)$ & $98(68-133)$ & 91 (72-114) \\
\hline CL: 25-75\% confidence limit & & & \\
\hline
\end{tabular}

Restenosis Rates after Stenting:

There were 933 patients who received a stent for treatment of stenosis at the cephalic arch with a subsequent follow-up encounter. During the 1 st index stent to 2 nd visit, these patients exhibited a median decrease of $16.5 \%$ per month in luminal diameter and had a median of 91 days between subsequent encounters (Table 2).

Within this group of patients who received a 1 st index stent, a subset of $5.1 \%$ and $0.5 \%$ of patients received a stent at the 2 nd index visit and 3rd index visit, respectively. These patients had a median decrease of $19.1 \%$ and $19.8 \%$ in luminal diameter per month and a median time of 91 and 95 days between encounters from the 2 nd index to 3 rd and 3 rd index to 4 th visits.

Assessment of stent types showed bare metal stents were more commonly used. At the 1 st index visit, $25.8 \%$ of stenting procedures used a stent graft and $74.0 \%$ used a bare metal stent (Table 4 ). Two patients did not have documentation of stent type and were not included. Stent grafts were used in $18.8 \%$ of stenting procedures at the 2 nd index visit and were never utilized at the $3 \mathrm{rd}$ index procedure. Between the 1st index to 2 nd visit, patients who received a bare metal stent showed a remarkably higher restenosis rate per month (10.1 percentage points higher) yet showed more days between encounters ( 5 more days) as compared to patients who received a stent graft. This difference in restenosis rates became smaller by stent types in the $2 \mathrm{nd}$ index to $3 \mathrm{rd}$ visit, where patients who received a stent graft had 5.4 percentage point lower restenosis rate per month and the same days between encounters, as compared to bare metal stents. Notably, there was only a select group of patients $(n=9)$ who received a stent graft at a $2 n d$ procedure. 
Table 4

Cephalic Arch Restenosis Rates Over Time by Stent Type

\begin{tabular}{|llll|}
\hline Bare Metal Stent & Visit Pair & & \\
\hline & 1st to 2nd & 2nd to 3rd & 3rd to 4th \\
\hline Change in Luminal Diameter & $\mathrm{n}=690$ & $\mathrm{n}=39$ & $\mathrm{n}=5$ \\
\hline Median \% Decrease per Month (CL) & $18.1(10.9-27.6)$ & $20.0(15.7-29.7)$ & 19.8 (13.0-24.6) \\
\hline Median Days between Encounters (CL) & $91(54-125)$ & $91(60-107)$ & $95(93-100)$ \\
\hline Stent Graft & Visit Pair & & \\
\hline & 1st to 2nd & 2nd to 3rd & 3rd to 4th \\
\hline Change in Luminal Diameter & $\mathrm{n}=241$ & $\mathrm{n}=9$ & $\mathrm{n}=0$ \\
\hline Median \% Decrease per Month (CL) & $8.0(0.0-23.6)$ & $14.6(0.0-18.8)$ & \\
\hline Median Days between Encounters (CL) & $86(29-130)$ & $91(58-133)$ & \\
\hline CL: $25-75 \%$ confidence limit & & & \\
\hline
\end{tabular}

\section{Restenosis Rates among Patients with an Index Stent and Subsequent Angioplasty}

Among the stent group defined at the 1 st index visit, $43.9 \%$ and $20.5 \%$ received an exclusive angioplasty without a stent at the subsequent 2 nd index and 3rd index visits, respectively (Table 5). Assessment of restenosis rates in the subsets of patients between the 2 nd index to $3 \mathrm{rd}$ and $3 \mathrm{rd}$ index to 4 th visits showed a decrease of $18.7 \%$ and $18.4 \%$ in the luminal diameter per month and a median time of 91 and 93 days between encounters. 
Table 5

Cephalic Arch Restenosis Rates Over Time among Patients with an Index Stent and Subsequent Angioplasty

\section{Any Stent Type at 1 st Index Visit, and Any Angioplasty Type at 2nd Index or 3rd Index Visits \\ Visit Pair}

2nd to 3rd 3rd to 4th

Change in Luminal Diameter

$n=410$

$18.7(11.9-$

25.4)

Median Days between Encounters (CL)

Median \% Decrease per Month (CL)

$91(68-$

119)

Visit Pair

Any Stent Type at 1 st Index Visit, and Standard Angioplasty at 2nd Index or 3rd Index Visits

\begin{tabular}{lll} 
& 2nd to 3rd & 3rd to 4 th \\
\hline Change in Luminal Diameter & $\mathrm{n}=337$ & $\mathrm{n}=155$ \\
\hline Median \% Decrease per Month $(C L)$ & $18.0(11.6-$ & $17.4(12.3-$ \\
& $25.1)$ & $23.3)$ \\
\hline Median Days between Encounters (CL) & $93(77-$ & $98(78-$ \\
& $121)$ & $121)$
\end{tabular}

Any Stent Type at 1st Index Visit, and High-Pressure Angioplasty at 2nd Visit Pair Index or 3rd Index Visits

\begin{tabular}{|c|c|c|}
\hline & 2nd to 3rd & 3rd to 4th \\
\hline Change in Luminal Diameter & $n=73$ & $n=36$ \\
\hline Median \% Decrease per Month (CL) & $\begin{array}{l}20.9(15.4- \\
26.9)\end{array}$ & $\begin{array}{l}19.7(14.4- \\
29.4)\end{array}$ \\
\hline Median Days between Encounters (CL) & $\begin{array}{l}91(63- \\
100)\end{array}$ & $\begin{array}{l}91(62- \\
106)\end{array}$ \\
\hline \multirow{2}{*}{$\begin{array}{l}\text { Bare Metal Stent at 1st Index Visit, and Any Angioplasty Type at 2nd } \\
\text { Index or 3rd Index Visits }\end{array}$} & Visit Pair & \\
\hline & 2nd to 3rd & 3rd to 4th \\
\hline Change in Luminal Diameter & $n=332$ & $n=149$ \\
\hline Median \% Decrease per Month (CL) & $\begin{array}{l}19.1(12.5- \\
25.4)^{(-5}\end{array}$ & $\begin{array}{l}18.4(13.3- \\
24.2)\end{array}$ \\
\hline Median Days between Encounters (CL) & $\begin{array}{l}91(70- \\
119)\end{array}$ & $\begin{array}{l}93(70- \\
119)\end{array}$ \\
\hline $\begin{array}{l}\text { Stent Graft at } 1 \text { st Index Visit, and Any Angioplasty Type at 2nd Index or } \\
\text { 3rd Index Visits }\end{array}$ & Visit Pair & \\
\hline
\end{tabular}




\begin{tabular}{|llll|}
\hline $\begin{array}{l}\text { Any Stent Type at 1st Index Visit, and Any Angioplasty Type at 2nd } \\
\text { Index or 3rd Index Visits }\end{array}$ & Visit Pair & \\
\hline Change in Luminal Diameter & 2nd to 3rd & 3rd to 4th \\
\hline Median \% Decrease per Month (CL) & $\mathrm{n}=76$ & $\mathrm{n}=42$ \\
\hline Median Days between Encounters (CL) & $16.1(10.7-$ & $17.6(11.3-$ \\
& $25.4)$ & $23.5)$ \\
\hline CL: 25-75\% confidence limit & $93(68-$ & $91(72-$ \\
\hline
\end{tabular}

Assessment by the angioplasty type used at the subsequent visits after an index stent procedure showed $17.8 \%$ and $18.8 \%$ of angioplasties used a high-pressure balloon at the 2 nd index and 3 rd index visits, respectively, and the remainder used a standard balloon. Restenosis rates were similar among patients who received a high-pressure angioplasty versus standard angioplasty and were consistent with restenosis rates among patients who never had a prior stenting procedure. Among patients who received a high-pressure angioplasty, median time between encounters was 2 and 7 days shorter between the 2nd index to 3rd and 3rd index to 4th visits, respectively, as compared to those who received a standard angioplasty.

Further assessment of restenosis rates associated with subsequent angioplasty procedures after an index stenting were performed by stent type. Subsequent angioplasties after a stent graft at the 1 st index visit were found to have a slightly lower restenosis rate per month (3 percentage points lower) between the $2 \mathrm{nd}$ index to $3 \mathrm{rd}$ visits with consistent days between encounters, as compared to bare metal stent. Restenosis rates and days were relatively similar between the 3rd index to 4th visits.

\section{Discussion:}

One of the most common, and easily addressed, complications with AVFs is development of primary and recurrent stenoses in various sites of the vascular access. [9] Each AVF type behaves differently as to where stenoses typically occur. Brachiocephalic AVFs (BCAVFs) are typically plagued by stenoses at the cephalic arch, with reports describing up to a $77 \%$ prevalence of significant stenoses at this site.[10] Angioplasty has been a mainstay of treatment for dialysis access stenoses, although literature has supported the use of stent graft placement compared with angioplasty alone.[11-14]

We found the luminal diameter at the cephalic arch decreased by $18.9 \%$ per month after the first exclusive angioplasty and $16.5 \%$ per month after the first stenting procedure among patients with a BCAVF. Restenosis rates were relatively similar between high-pressure and standard angioplasty procedures, suggesting consistent effectiveness when a high-pressure balloon was required to eliminate the lesion. Consistent with the literature,[13] restenosis rates were higher after the first bare metal stent $(18.1 \%$ per month) compared to stent graft ( $8.0 \%$ per month). Overall, these findings indicate that once a patient 
presents with a cephalic arch stenosis and is treated with an angioplasty or stent, they are at risk of having a $50 \%$ stenosis in 2.65 or 3.03 months, respectively. Stated differently, a patient with a post intervention residual stenosis of $10 \%$ after their first exclusive angioplasty or stent is estimated to progress to have a $67 \%$ or $60 \%$ stenosis in 3 months, respectively. Restenosis rates were relatively similar across subsequent angioplasty or stent procedures during follow-up.

One of the longest retrospective studies involving hemodialysis upper arm AVFs found a 34\% primary and $82 \%$ secondary patency rate at one year, although there was no differentiation between brachiocephalic versus brachiobasilic fistulae.[6] This study found the interval for re-intervention was 10.6 months in upper arm AVFs, but did not specify the exclusivity or combinations of intervention types nor the site of malfunction. We observed the interval for re-intervention after exclusive angioplasty or stenting procedures was approximately 3 months. However, the confidence limits for restenosis rates and reintervention times were typically large, which represents high patient-to-patient variability.

Future studies are needed to identify the predictors of restenosis risk at the cephalic arch. Arterialized pressures, intimal hyperplasia, and turbulence are likely culprits for rapidly recurrent and possibly angioplasty-resistant stenoses.[15] Fistula age may also be affecting the durability of the vessel wall.[6] Neo-intimal hyperplasia and turbulence has been suggested as a cause of rapid recurrent in-stent stenosis. $[11,16,17]$ For bare metal stents, the intima continues to be exposed to turbulent flow and biochemical constituents of blood vessels through the interstices,[17] and may explain why lower restenosis rates were seen for stent grafts. Although not evaluated, several incidences of stent fracture were noted as causing recurrent stenosis; oftentimes, angioplasty and/or stenting was required to improve the flow and appearance.

Our study has several strengths, the most obvious of which includes the focus on exclusive interventions that afforded the ability to reasonably assess restenosis rates at a specific lesion site. In addition, the analysis included a large number of patients treated by various practitioner types in a broad geography. All these factors represent clinically relevant interventions and consequently, this data likely represents reproducible, real world outcomes. However, this study cohort may not be fully generalizable and is not representative of the groups of patients who had unremarkable complications upon evaluation, or a mixture of interventions (e.g. thrombectomy and high-pressure angioplasty). Also, drug eluting balloons were not evaluated due to many factors, including minimal adoption in the outpatient setting that is likely secondary to cost and familiarity with the equipment.

The retrospective nature of this study is an inherent weakness. Also, stenosis measurements may have varied by practitioner, although a small random sampling of cases with image review showed good agreement with reported degrees of stenosis. Given the large number of centers, inter-operator variability also poses a problem when evaluating the need for stenting, stent type, and general personal practice regarding stenting versus angioplasty alone. Also, the precise morphology of the stenosis, focal versus long segment versus multifocal, was not clearly specified. While morphology may play a role in the longterm success of endoluminal interventions, it is not clear stratifying the results based on morphology 
would have any impact. Lastly, these models of recurrence are based on a linear progression of stenosis development warranting treatment. It is feasible that after numerous interventions, the rate of progression may change, either for better or worse.

\section{Conclusions:}

In conclusion, the study demonstrated restenosis rates at the cephalic arch in hemodialysis patients with a brachiocephalic fistula requiring an angioplasty or stent were $18.9 \%$ and $16.5 \%$ per month after the first intervention, respectively. Restenosis rates appeared to be relatively consistent across subsequent procedures. Findings suggest patients are at risk of having significant lesions ( $>50 \%$ stenosis) within 3 months after the previous intervention at the cephalic arch. When clinically indicated, use of stent grafts seemed to lessen recurrent stenosis, especially if used as the first stent. It appears prudent to have patients who develop a cephalic arch lesion return for an evaluation after approximately 3 months. At that time, a careful history and physical exam should be performed to determine if recurrence is present. If the assessment indicates such, imaging with possible intervention is recommended to maximize patency and functionality of a patient's hemodialysis access, thereby prolonging life.

\section{List Of Abbreviations:}

ATM: atmospheres

AVF: arteriovenous fistula

AVG: arteriovenous graft

BCAVF: brachial artery to cephalic vein arteriovenous fistula

IRB: Independent Review Board

KRT: kidney replacement therapy

\section{Declarations:}

\section{Ethics Approval and Consent to Participate:}

This analysis was conducted under a protocol that was reviewed and approved by New England Independent Review Board (IRB) (Needham Heights, MA, United States; NEIRB\#WO-1-574-1). The IRB approved the protocol under an Exempt Category since the analysis used deidentified patient data that did not include Protected Health Information and written/verbal informed consent was not required nor applicable per the Code of Federal Regulations(45CFR46) in the United States. This study adhered with the Declaration of Helsinki. 
Not applicable.

\section{Availability of Data and Materials:}

The dataset used for this analysis is not publicly available. The data utilized was obtained from a private electronic medical record system, which is restricted to use by only authorized employees. Reasonable requests to access the study dataset might be considered under executed contractual agreements between Fresenius Medical Care and an external individual's institution. Requests to access the dataset can be sent to the author J.W.L.

\section{Competing interests:}

All authors are employees of Fresenius Medical Care, or its subsidiary company Azura Vascular Care. L.A.U. has share options/ownership in Fresenius Medical Care. L.A.U., J.W.L. are an inventor on patent(s) in the field of dialysis. J.W.L. is a guest editor on the Editorial Board of Frontiers in Physiology.

\section{Funding:}

The analysis and manuscript composition were internally supported by Fresenius Medical Care.

\section{Authors' Contributions:}

Research idea and study design: R.N.R., M.R., Y.J., L.A.U., and J.W.L.; data acquisition: R.R., M.R., and N.M.; data analysis: Y.J. and L.A.U.; interpretation: R.N.R., M.R., Y.J., L.A.U., M.S., and J.W.L.; supervision or mentorship: R.N.R., L.A.U., M.S., and J.W.L.; All authors contributed to the manuscript drafting and/or revision.

\section{Acknowledgements:}

This article is in honor of the contributing author Melvin "Mel" Rosenblatt who passed away during the finalization of the analysis and manuscript composition. Mel was an impetus to this study and majorly contributed to the initial draft of this manuscript. Also, we would like to thank the interventionalists and care teams at Azura Vascular Care who captured the data used in this analysis during the provision of standard medical care.

\section{References}

1. United States Renal Data System. 2020 USRDS Annual Data Report: Epidemiology of kidney disease in the United States. In. National Institutes of Health, National Institute of Diabetes and Digestive and Kidney Diseases, Bethesda, MD.

2. Lok CE: Fistula first initiative: advantages and pitfalls. Clin J Am Soc Nephrol 2007, 2(5):1043-1053.

3. Quencer KB, Oklu R: Hemodialysis access thrombosis. Cardiovasc Diagn Ther 2017, 7(Suppl 3):S299S308. 
4. Quencer KB, Arici M: Arteriovenous Fistulas and Their Characteristic Sites of Stenosis. American Journal of Roentgenology 2015, 205(4):726-734.

5. Rajan DK, Bunston S, Misra S, Pinto R, Lok CE: Dysfunctional autogenous hemodialysis fistulas: outcomes after angioplasty-are there clinical predictors of patency? Radiology 2004, 232(2):508515.

6. Turmel-Rodrigues L, Pengloan J, Baudin S, Testou D, Abaza M, Dahdah G, Mouton A, Blanchard D: Treatment of stenosis and thrombosis in haemodialysis fistulas and grafts by interventional radiology. Nephrol Dial Transplant 2000, 15(12):2029-2036.

7. Rajan DK, Clark TW, Patel NK, Stavropoulos SW, Simons ME: Prevalence and treatment of cephalic arch stenosis in dysfunctional autogenous hemodialysis fistulas. J Vasc Interv Radio/ 2003, 14(5):567-573.

8. Kian K, Asif A: Cephalic arch stenosis. Semin Dial 2008, 21(1):78-82.

9. Campos RP, Do Nascimento MM, Chula DC, Do Nascimento DE, Riella MC: Stenosis in hemodialysis arteriovenous fistula: evaluation and treatment. Hemodial Int 2006, 10(2):152-161.

10. Hammes M, Funaki B, Coe FL: Cephalic arch stenosis in patients with fistula access for hemodialysis: relationship to diabetes and thrombosis. Hemodial Int 2008, 12(1):85-89.

11. Shemesh D, Goldin I, Zaghal I, Berlowitz D, Raveh D, Olsha O: Angioplasty with stent graft versus bare stent for recurrent cephalic arch stenosis in autogenous arteriovenous access for hemodialysis: a prospective randomized clinical trial. J Vasc Surg 2008, 48(6):1524-1531, 1531 e1521-1522.

12. Rajan DK, Falk A: A Randomized Prospective Study Comparing Outcomes of Angioplasty versus VIABAHN Stent-Graft Placement for Cephalic Arch Stenosis in Dysfunctional Hemodialysis Accesses. $J$ Vasc Interv Radio/ 2015, 26(9):1355-1361.

13. D'Cruz R T, Leong SW, Syn N, Tiwari A, Sannasi VV, Singh Sidhu HR, Tang TY: Endovascular treatment of cephalic arch stenosis in brachiocephalic arteriovenous fistulas: A systematic review and meta-analysis. J Vasc Access 2019, 20(4):345-355.

14. Miller GA, Preddie DC, Savransky Y, Spergel LM: Use of the Viabahn stent graft for the treatment of recurrent cephalic arch stenosis in hemodialysis accesses. J Vasc Surg 2018, 67(2):522-528.

15. Faxon DP, Weber VJ, Haudenschild C, Gottsman SB, McGovern WA, Ryan TJ: Acute effects of transluminal angioplasty in three experimental models of atherosclerosis. Arteriosclerosis 1982, 2(2):125-133.

16. Roy-Chaudhury P, Arend L, Zhang J, Krishnamoorthy M, Wang Y, Banerjee R, Samaha A, Munda R: Neointimal hyperplasia in early arteriovenous fistula failure. Am J Kidney Dis 2007, 50(5):782-790.

17. Sivananthan G, Menashe L, Halin NJ: Cephalic arch stenosis in dialysis patients: review of clinical relevance, anatomy, current theories on etiology and management. J Vasc Access 2014, 15(3):157162.

\section{Figures}




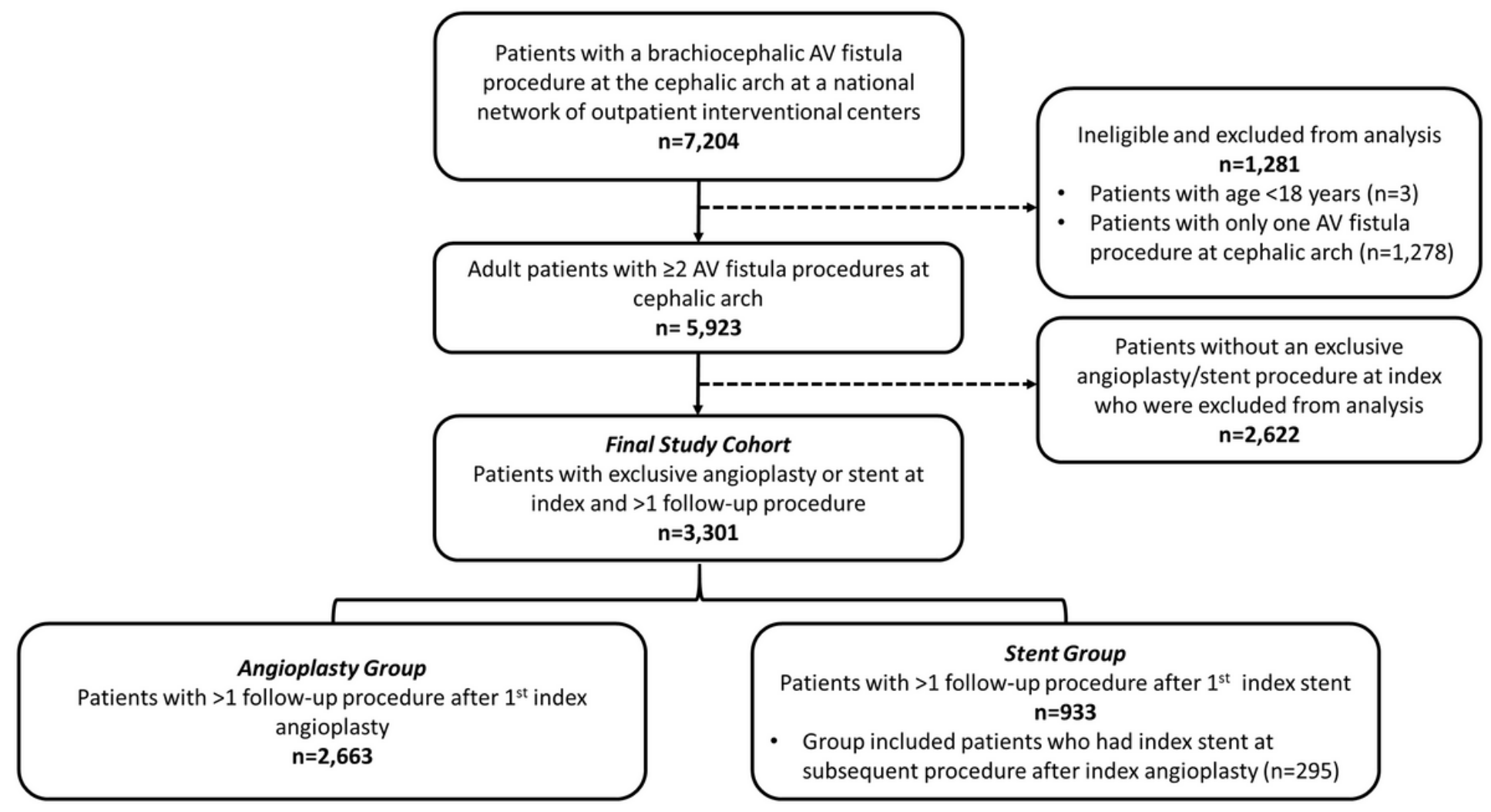

Figure 1

Patient Flow Diagram 